\title{
Research on Method of Trajectory Prediction in Aircraft Flight Based on Aircraft Performance and Historical Track Data
}

\author{
Shu-Yuan Jiang, ${ }^{1,2}$ Xiling Luo $\mathbb{D}^{2,3}$ and Liang $\mathrm{He}^{1}$ \\ ${ }^{1}$ Nanjing Les Information Technology Co., Ltd., Nanjing 210014, China \\ ${ }^{2}$ Hangzhou Innovation Institute, Beihang University, Hangzhou 310052, China \\ ${ }^{3}$ College of Electronic Information Engineering, Beihang University, Beijing 100191, China \\ Correspondence should be addressed to Xiling Luo; 499566293@qq.com
}

Received 24 November 2020; Revised 2 February 2021; Accepted 9 February 2021; Published 27 February 2021

Academic Editor: Kamal Shah

Copyright (c) 2021 Shu-Yuan Jiang et al. This is an open access article distributed under the Creative Commons Attribution License, which permits unrestricted use, distribution, and reproduction in any medium, provided the original work is properly cited.

\begin{abstract}
Traditional 4D trajectory prediction based on aircraft performance models and flight procedures does not consider control handover rules. Meanwhile, method based on historical data mining cannot accurately couple with real-time conditions such as weather and also cause computational efficiency problems. This project collected a large amount of historical data to form a control experience database and mined the historical database to obtain control experience and flight intention. On the basis of the traditional aircraft performance model, this paper puts forward the aircraft maneuver mode using strategy and introduces the high-altitude wind information from the weather information into the aircraft $4 \mathrm{D}$ model to optimize the aircraft $4 \mathrm{D}$ trajectory calculation model. By comparing the flight forecast time with the real crossing time, it is found that the average error of the improved $4 \mathrm{D}$ forecast crossing time is less than $5 \%$ of the flight time, which is obviously better than that before optimization. It is proved that the optimized method based on historical track data is effective and reliable, and the accuracy of $4 \mathrm{D}$ track prediction is improved greatly.
\end{abstract}

\section{Introduction}

With the development of civil aviation industry, there are more and more aircraft on the flight path, the flight spacing is smaller and smaller, and the air traffic safety situation is more and more serious. Flight delays not only bring direct economic losses to passengers and airlines but also affect the development of the national economy. In recent years, automatic and intelligent methods of air traffic control [1], such as aircraft conflict detection and resolution [2], sequence of approach and departure [3], and trajectory-based operation [4], have become increasingly popular. The purpose of these methods is to improve air traffic efficiency and ensure air traffic safety [5]. The rapid and accurate aircraft trajectory prediction is the basis and guarantee for the realization of the above methods [6].

The 4D (4-dimensional) track prediction is to predict and calculate the $4 \mathrm{D}$ track points that will be generated by a flight according to the experience information and initial plan information when a flight does not occur. At present, there are two main methods for aircraft $4 \mathrm{D}$ track prediction [7-10]. One is to synthesize all kinds of information, such as type, position, altitude, speed of the departure airport, landing airport, and reporting point in the plan information, the aircraft standard flight procedures, the aircraft cruising altitude and speed, and the conversion altitude, to form a horizontal trajectory, an altitude trajectory, and a velocity trajectory by using the aircraft performance model. In this process, taking into account the influence of the high-altitude wind on the ground speed of the aircraft, the three profiles are processed independently and coupled to calculate the $4 \mathrm{D}$ trajectory of the aircraft. The position, speed, height, and control sector of the flight passing through each reporting point can be obtained. For example, in [11], a method of predicting $4 \mathrm{D}$ taxiing trajectory for aircraft on airport surface based on fitting velocity profile is proposed. In [12], Schuster builds on an existing en route trajectory prediction (TP) 
model and develops novel techniques to predict aircraft trajectories for the transitions between the ground and en route phases of operation and the ground phase. The remaining parameters were taken from the BADA (Base of Aircraft Date) 3.7 model. Du et al. [13] put forward 7 kinds of track segment models based on the route object method and built the horizontal track according to the geographical distribution of flight plan, control instruction, and navigation equipment, and the important parameters (climb rate, drop rate, thrust, cruise Mach number, etc.) needed for vertical profile modeling are simply calculated and optimized. Michael Kaiser et al. present an analytical model of the aircraft performance and apply the findings to precisely predict fuel flow characteristics as fundamental parameter for trajectory planning [14]. And the presented enhanced jet performance model provides a significant improvement over the currently applied trajectory prediction method. An aircraft performance model has been constructed based on BADA 4 so as to generate trajectories in [15], and the multimodal approach for the aircraft model is utilized to overcome the problem of the complex optimal trajectory generation via reducing the dimension.

The other track prediction method is based on track data mining with the rise of big data technology. Historical track data are a reliable record of past flight conditions, which include all possible factors affecting the operation of an aircraft, such as changes in flight plans, control rules and experience, and weather conditions. This information can be mined to analyze track patterns and used in track prediction to improve prediction accuracy. For example, Zhao et al. proposed a deep long short-term memory (D-LSTM) neural network for aircraft trajectory prediction, which improves the prediction accuracy of aircraft in complex flight environments [16]. Zeng et al. [17] formulated the 4D trajectory prediction problem as a sequence-to-sequence learning problem and proposed a sequence-to-sequence deep long short-term memory network (SS-DLSTM) for trajectory prediction. Gabriel et al. take turning points as clustering objects and use the clustering method to sort out the track data and exclude the departure track to extract the typical track, which is used for aircraft surveillance and prediction, but the track information loss is more, and there is a lack of altitude and time information [18]. Zhao et al. [19] proposed a density-based improved track clustering method for track prediction by combining weighted Manhattan distance with penalty coefficient. Qian et al. [20] put forward an air target track prediction model based on reverse propagation neural network, which can cluster the target track data adaptively and extract the rule of specific target's moving area.

The first $4 \mathrm{D}$ trajectory prediction method based on the aircraft performance model is difficult to guarantee the accuracy of trajectory prediction without considering the weather model or judging the actual intention of aircraft. For example, the transfer of aircraft between different control areas is subject to the relevant protocol constraints of the control transfer provisions, and it is difficult to take into account such control transfer rules and altitude variation characteristics in standard flight procedures. This will cause more obvious forecast error. The second track prediction algorithm based on big data technology fully exploits flight history characteristics, but it is not easy to integrate other factors that affect flight, such as real-time weather and temporary change of flight path. On the other hand, the large amount of air transportation data, multidata types, and various data distribution characteristics will lead to low efficiency in the actual track prediction process and cannot meet the real-time requirements. In addition, the abnormal track has a great influence on the generation of the representative track, and it also has an impact on the prediction.

In order to solve this problem, this paper will make full use of the advantages of aircraft performance models and consider obtaining empirical parameters from historical track data (for example, reporting point-to-point altitude) to reflect the actual control intention, and the coupling calculation method of the two is designed. At the same time, taking into account the influence of high-altitude wind, the $4 \mathrm{D}$ track prediction accuracy can be improved, and the prediction results are compared with the actual radar track data to verify the effectiveness of the method.

\section{Historical Data Analysis}

In this paper, the historical track data are collected for a period of time, and the acquired track data are fused, the cleaned data are matched with the corresponding flight plan data, and the flight record table of the aircraft is established. Each flight record is composed of aircraft type, flight number, route, planned takeoff time, planned landing time, actual takeoff time, actual landing time, departure airport, destination airport, track point, latitude and longitude, transit time, transit speed, transit altitude, and other attributes. Delete the invalid record that the flight number is empty, add the field of the serial number, and sort by the transit time from the departure time to the landing time of a flight. The flight number, aircraft type, serial number, actual departure time, departure airport, destination airport, track point, latitude and longitude, transit time, transit velocity, and transit altitude are migrated to the distributed database HBase of the Hadoop cluster. The GRIB (Gridded Binary) meteorological forecast data of the same period are analyzed and put into the database to provide the necessary meteorological forecast information for data mining.

Then, the first step is to get the actual cross-point information of the reporting point. According to the route start-up time, the national route model database is established, which mainly contains 5 attributes, namely, route number, start-up time, end-up time, route reporting point, and latitude and longitude. The mapping model of data file is constructed by using flight record (associated field route) and national route model database (associated field route number). Map raw data are stored in HBase to intermediate data containing only route reporting points and track points. By using the distance formula between the two points, the report points and the track points which are less than a certain threshold $(20 \mathrm{~km})$ are temporarily stored. Take the minimum distance of the track point to replace the reporting point, and the track point crossing point height is the reporting point crossing point height. 
The second step is to get the altitude levels of the reporting point and the track point. According to the standard of RVSM airspace in China, a parameter database of altitude levels is established, which mainly contains three attributes: altitude level sequence number, low-altitude level, and high-altitude level. The mapping model of the data file is constructed by using the national route model database (association fields are the low-altitude level and high-altitude level), and the original altitude data of the reporting point and the track point are mapped to the altitude levels.

Using the altitude levels obtained by the above steps, the experience altitude level of each reporting point under the condition of the same airport, the same aircraft type, and the same route is obtained by using $\mathrm{k}$-means hard clustering algorithm and then mapped to the height of the altitude layer.

$\mathrm{K}$-means clustering is also known as fast clustering or dynamic clustering. Before clustering, it is necessary to determine the classification number $k$ according to the specific problem and divide the data into $k$ clusters according to the distance function through the iterative process, so as to optimize the criterion function for evaluating the clustering performance [21].

Suppose that the observation sample $X$ contains $n$ sample points, $X=\left\{x_{1}, x_{2}, \ldots, x_{n}\right\}$. In this paper, Euclidean distance (4) is used to evaluate the similarity:

$$
\mathrm{d}\left(x_{i}, x_{j}\right)=\left[\sum_{k=1}^{w}\left(x_{i k}-x_{j k}\right)^{2}\right]^{(1 / 2)} .
$$

The k-means algorithm uses the sum of square error criterion function to evaluate the clustering performance. Suppose that $X$ contains $k$ clustering subsets $X_{1}, X_{2}, \ldots, X_{\mathrm{k}}$, and the number of sample points of each subset is $n_{1}, n_{2}, \ldots$, $n_{\mathrm{k}}$, the mean value of each subset is $m_{1}, m_{2}, \ldots, m_{\mathrm{k}}$, and then the formula of the criterion function of the sum of squares of errors is as follows:

$$
E=\sum_{i=1}^{k} \sum_{p \in X_{i}}\|p-m\|^{2} .
$$

On the other hand, using the altitude level information of the track points and the passing time of the track points in the flight record as the basic information to calculate the cruise altitude, the information of the flight time lasting below a certain range (10 minutes) is deleted. The altitude level of each flight with the longest duration is then obtained as the cruising altitude. By coupling the track point ground velocity of the longest time with the historical high-altitude wind velocity at the position of the track point, the vacuum velocity of each track point is obtained. Then, the cruise phase vacuum velocity is obtained using the mean method.

Data information obtained from the above calculation process is recorded, and the route experience data information table is established. The specific record information includes aircraft type, flight number, departure airport, destination airport, name of reporting point, altitude level of reporting point, cruising altitude, and route.

\section{Flight Profile Modeling Based on Aircraft Performance}

For the received, created, modified, or extracted flight plan processing information, the experienced information of the reporting point in the route experience information table obtained in Section 2 is used to replace the report point height and cruise altitude in the flight plan. Combined with the meteorological forecast information of the received period, a 4D track prediction model based on the control intent and aircraft performance model is constructed.

According to the characteristics of flight phase, $4 \mathrm{D}$ predicted track is divided into three sections: horizontal profile, altitude profile, and velocity profile, as shown in Figure 1. Modeling idea: firstly, synthesize the two-dimensional horizontal flight path from the starting point to the end point, and then study the velocity profile and altitude profile along the known horizontal trajectory, so as to realize the $4 \mathrm{D}$ track simulation calculation.

3.1. Horizontal Flight Profile. From the horizontal track of a typical flight trajectory, an aircraft usually flies in a straight line from one point to another, then turns around this point or near it and enters a new course, and then flies in a straight line again. Therefore, the horizontal motion of aircraft mainly includes linear motion model and turning motion model [22]. Aiming at the turning motion, this paper adopts the internal shear turning model. As shown in Figure 2, the turning radius is $R=V_{\mathrm{Gs}} /(g \sin \varphi)$ and the turning inclination angle is $\varphi$. For the takeoff stage, the general value is about $15^{\circ}$. The aircraft flies directly from point $\mathrm{P} 1$ to point $\mathrm{P} 2$, turns at point $\mathrm{P}$ with turning radius $\mathrm{R} 2$, and intercepts the new course at point $\mathrm{Q}$. The longitude and latitude coordinates of P1, P2, and P3 points were known. Cartesian coordinates $\left(x_{P i}, y_{P i}\right)(i=1,2, \ldots)$ were obtained by coordinate transformation.

The course is

$$
\begin{array}{ll}
\psi_{2}=\tan ^{-1}\left(\frac{y_{P 2}-y_{P 1}}{x_{P 2}-x_{P 1}}\right), & -\pi \leq \psi_{2} \leq \pi, \\
\psi_{3}=\tan ^{-1}\left(\frac{y_{P 3}-y_{P 2}}{x_{P 3}-x_{P 2}}\right), & -\pi \leq \psi_{3} \leq \pi .
\end{array}
$$

According to the course of the two direct sections, the turning angle of the aircraft can be obtained:

$$
\Delta \psi= \begin{cases}\psi_{3}-\psi_{2}+2 \pi, & \text { SIGN }>0 \text { and } \psi_{3}<\psi_{2}, \\ \psi_{3}-\psi_{2}-2 \pi, & \text { SIGN }<0 \text { and } \psi_{3}>\psi_{2}, \\ \psi_{3}-\psi_{2}, & \text { others. }\end{cases}
$$

$\operatorname{SGN}(\Delta)$ function value is as follows: when $\Delta>0$ and $\operatorname{SGN}(\Delta)=1$, turn right; when $\Delta=0$ and $\operatorname{SGN}(\Delta)=0$, go straight; when $\Delta<0$ and $\operatorname{SGN}(\Delta)=-1$, turn left.

According to turning angles $\Delta \psi$ and turning radius $R$, we can get $C_{2}$, the flight distance, turning distance, and the coordinate position of turning starting point and ending point. 


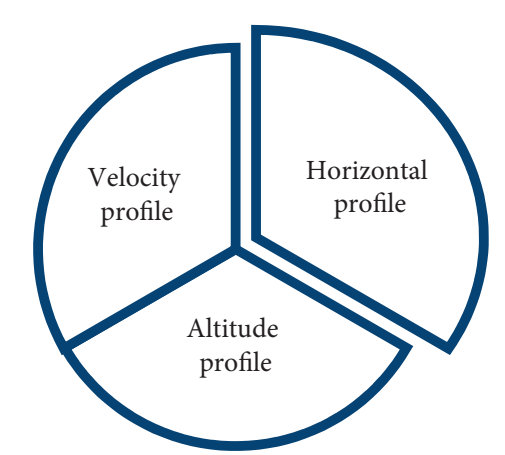

FiguRE 1: Schematic diagram of aircraft trajectory profile.

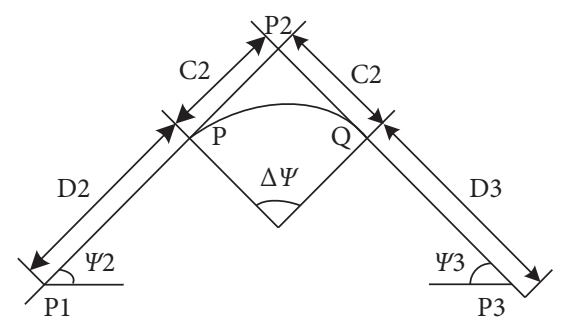

FIgURE 2: Schematic diagram of internal shear turning model.

3.2. Height and Velocity Profile. In the process of building an aircraft flight model, under no special conditions, the default flight path is to climb to the cruising altitude first and then fly a distance at the cruising altitude in accordance with the standard flight procedures, and finally, follow the standard descent procedure to land at the airport. For example, the ascent phase can be modeled as shown in Figure 3. And the descent phase can be modeled as shown in Figure 4.

3.2.1. Aircraft Performance Model. For each stage, the laws of speed and altitude are solved based on the aircraft performance model. BADA is an aircraft performance database developed by the French EC (EuroControl) Center, which contains different types of aircraft performance parameters and operating program parameters $[12,15]$. BADA is based on the TEM (Total Energy Model) model of the aircraft, and the aircraft is modeled as a particle. The force acting on the aircraft is shown in Figure 5. The work done by the resultant force acting on the aircraft is equal to the kinetic energy and potential energy increment of the aircraft:

$$
m \frac{\mathrm{d} V_{\mathrm{Tas}}}{\mathrm{d} t}=(T-D)+m g \sin \gamma
$$

where $m$ is the aircraft mass; $V_{\text {Tas }}$ is the vacuum speed; $T$ is the thrust; $D$ is the resistance; $g$ is the gravitational acceleration; and $\gamma$ is the aircraft climb/descent angle.

The rate of increase and decrease is as follows:

$$
\frac{\mathrm{d} h}{\mathrm{~d} t}=-V_{\mathrm{Tas}} \sin \gamma=\frac{(T-D) V_{\mathrm{Tas}}}{m g}\left(1+\frac{V_{\mathrm{Tas}}}{g} \frac{\mathrm{d} V_{\mathrm{Tas}}}{\mathrm{d} h}\right)^{-1}
$$

where $h$ is the height. $\left(1+\left(V_{\text {Tas }} / g\right)\left(\mathrm{d} V_{\text {Tas }} / \mathrm{d} h\right)\right)^{-1}$ is the energy distribution coefficient. It can be transformed into a function of Mach number $f(M)$, representing the ratio of the thrust used for climbing to the thrust used for acceleration when climbing at a selected speed. The method is as described in [22].

3.2.2. Processing of Meteorological Conditions. The influence of meteorological factors on flight path prediction was studied by using the high-altitude wind information of the GRIB meteorological report [15]. The GRIB weather report is published by WAFS (World Area Forecast System). According to the need of calculation, the high-altitude wind/ temperature data in GRIB were analyzed and interpolated, and the high-altitude wind speed, wind direction, and temperature of different latitude and longitude and altitude layer grids were given. According to the position and altitude information of the predicted route, the corresponding highaltitude wind $\left(\vec{V}_{\text {wind }}\right)$ was invoked and the corresponding ground velocity $\left(\vec{V}_{\mathrm{GS}}\right)$ was obtained by vector calculation with the calculated vacuum velocity $\left(\vec{V}_{\text {TAS }}\right)$, as shown in Figure 6, and thus, the track prediction results were corrected. Similarly, for the cruise velocity obtained in Section 2 , the vacuum velocity is obtained by calculating the track point ground velocity and the wind velocity vector.

3.3. Profile Coupling. Based on the information of the planned distance and the location of the initial reporting points, the geographic coordinates of the predicted trajectory points are calculated, and the corresponding velocity and altitude results in the velocity and altitude profiles are 


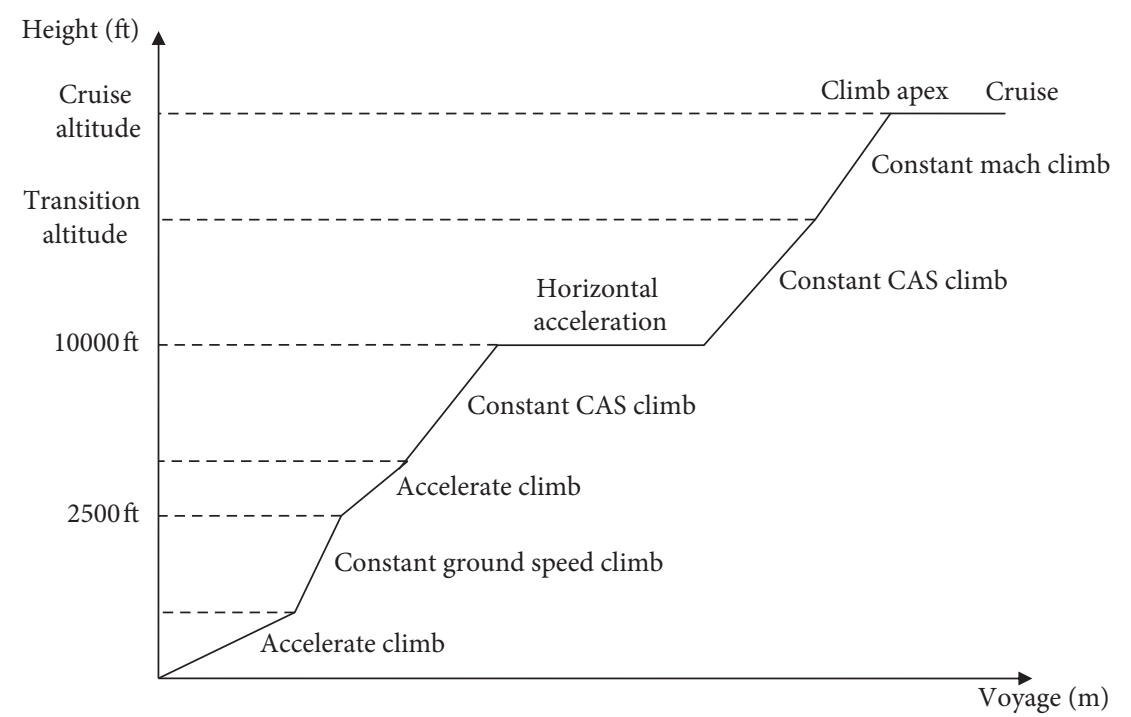

Figure 3: Schematic diagram of aircraft standard ascent phase.

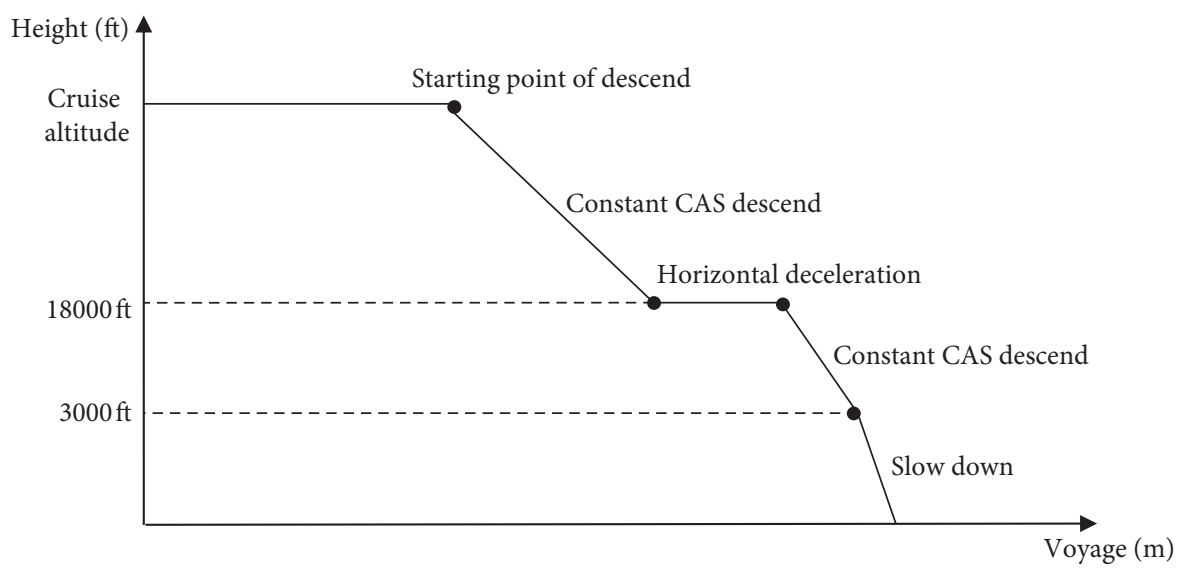

FIGURE 4: Schematic diagram of aircraft standard descent phase.

obtained by considering the distance. Finally, the predicted passing time, passing height, and location coordinates of all the trajectory points and the reporting points are obtained.

\section{Correction Method with Historical Empirical Parameters}

In the actual flight process, the aircraft needs to accept different control commands when passing through different control areas. On the basis of sector handover protocol, it is necessary to command the aircraft to enter the designated flight level and hand over to the next control sector through altitude adjustment. The height restriction requirements between different sectors will affect the height of aircraft arriving at each reporting point. In the calculation of track prediction, the experienced point height of each report point obtained from the historical track mining is taken as the target altitude, and aircraft are required to follow each specified altitude when passing through each report point. Based on the standard flight procedure, we propose to divide the flight process from one reporting point 1 to another reporting point 2 into three modes for calculation, as shown in Figure 7.

Mode 1: the aircraft climbs/descends from the altitude of report point $\mathrm{P} 1$ to the altitude of report point $\mathrm{P} 2$, and the climbing/descending attitude matches the aircraft performance model. After reaching the altitude of reporting point P2, it flies horizontally to report point P2; Mode 2: the aircraft flies horizontally for a distance at the altitude of reporting point $\mathrm{P} 1$ and then climbs/descends from the altitude of reporting point $\mathrm{P} 1$ to the altitude of reporting point P2. The climbing/descending attitude matches the aircraft performance model; Mode 3: the aircraft climbs from the altitude of report point P1 to cruise altitude and then climbs/ descends from cruise altitude to report point P2. The climb/ descent attitude matches the aircraft performance model.

According to the position of the current computing point and the next computing point in the total route and the position between the two points, the three predictive flight modes are chosen. We designed the following method of use: 


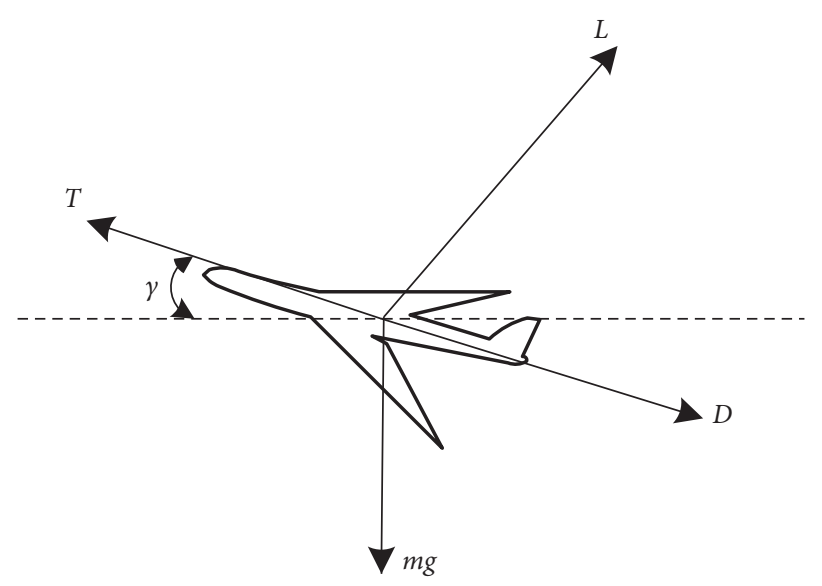

FIgURE 5: Schematic diagram of aircraft stress status.

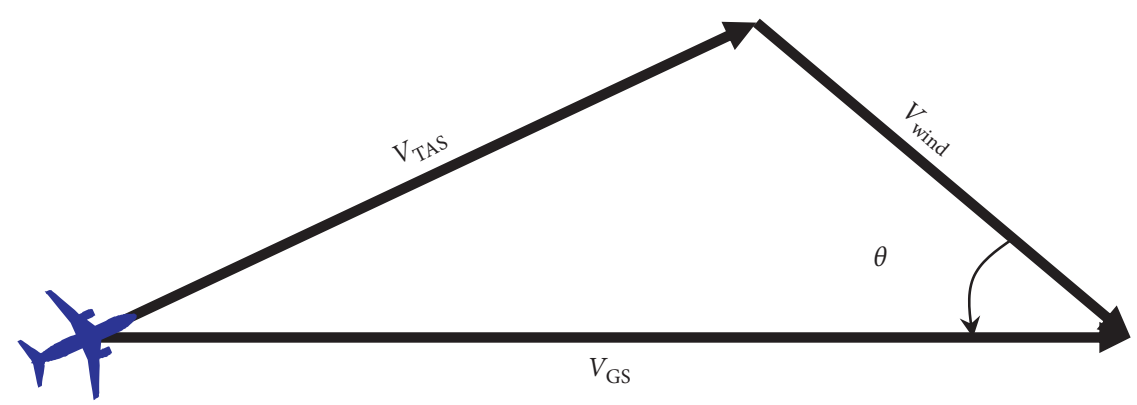

FIgURE 6: Schematic diagram of velocity calculation.

Step 1: first, assume that the current compute point distance is $S_{0}$. Based on aircraft performance models and standard flight procedures, the distance required to climb/descend to the next altitude at the current compute point is estimated to be $S_{1}$. The total track target distance is $S_{\text {total }}$. The distance required for departure airport to climb to cruising altitude is $S_{\text {climb }}$ and the distance required for cruising altitude to descend to destination airport is $S_{\text {descend }}$.

Step 2: when the current calculated point height is less than the cruising altitude, the flight range over a certain threshold $S_{\mathrm{m}}$, there is still the possibility of entering the cruising altitude. Our design is as follows: when the next computing point with the target height is the destination airport, choose Mode 3; when the next computing point with the target height is not the destination airport, and the distance to the next point is far, while leaving enough range for descent, select Mode 1; otherwise, select Mode 3. For example, when $S_{\text {total }}>=n_{1}{ }^{*} S_{\text {climb }}+n_{2}{ }^{*} S_{\text {descend }}$, take $S_{m}=n_{1}{ }^{*} S_{\text {climb }}$, when $\quad S_{\text {total }}>=n_{1}{ }^{*} S_{\text {climb }}+n_{2}{ }^{*} S_{\text {descend }}$, take $S_{m}=0.5^{*} S_{0}$, and $n_{1}, n_{2}$ are adjustable coefficients, take $n_{1}>1$ and $n_{2}>1$. If $n_{2}{ }^{*} S_{\text {descend }}>S_{0}+S_{1}>n_{1}{ }^{*} S_{\text {climb }}$, then choose Mode 1; otherwise, select Mode 3.

Step 3: if the condition of Step 2 is not met and the current point is higher than the cruising altitude or has already flown a longer distance, the possibility of climbing to the cruising altitude is no longer considered. When the next computing point with the target height is the destination airport, select Mode 2. When the distance from the current computing point to the next computing point is less than $n_{3}{ }^{*} s_{1}$, select Mode 1.When the distance from the current computing point to the next computing point is more than $n_{4}{ }^{*} s_{1}$, select Mode $2 ; n_{3}$ and $n_{4}$ are adjustable coefficients.

\section{Numerical Simulation and Verification}

In accordance with the above steps, the historical track and flight plan data of North China over a period are collected, and the statistics are made according to city pairs and aircraft types. The experienced altitude, the experienced cruise altitude, and the experienced cruise velocity data of the flight reporting points under the constraints of urban pair and aircraft type are obtained and provided to the track prediction module as configuration parameters.

The track prediction module first synthesizes the information such as the departure airport, landing airport, and waypoint in the real flight plan information to form a horizontal trajectory. Then, according to the standard flight procedure of the aircraft, the planned or experienced cruise altitude and cruise speed of the aircraft, the converted 
altitude, and the altitude or experienced altitude of the reporting point, it generates an altitude profile and a velocity profile which can be divided into several sections. Together with the horizontal profile, the $4 \mathrm{D}$ trajectory of the aircraft is synthesized.

Taking a B77w flight from Shenyang (ZJSY) to Beijing (ZBAA) airport as an example, through historical data mining and analysis, the empirical information is obtained as shown in Table 1. It should be noted that since the data are collected in North China, there is no effective historical track data collected in a period before entering the North China intelligence region. Therefore, the empirical value obtained by data mining mainly focuses on the report points after entering the North China intelligence region. In addition, some waypoints do not obtain effective experience information due to data divergence or small amount of data. In this paper, only empirical information with high degree of data aggregation is used as prediction parameters.

The experiential altitude of each report point is used as the target altitude, and the experiential cruise altitude and cruise speed are used to replace the cruise altitude and speed information in the plan for the $4 \mathrm{D}$ trajectory prediction. Figures 8-10 show the comparison between the predicted trajectory and the actual trajectory of an A320 flight from Shenyang to Beijing airport on October 26, 2019. Among them, the prediction result obtained when the control experience altitude obtained by big data analysis is not introduced is represented by gray, the prediction curve obtained by introducing big data analysis result is shown in blue, and the actual flight trajectory received by radar is represented by orange. It can be seen from the three graphs that the accuracy of the prediction track and the real track is greatly improved after adding the empirical altitude and speed information obtained from historical track analysis, which shows that the modeling and calculation process in this paper is accurate. The track prediction algorithm based on the combination of control intention and aircraft performance can greatly improve the accuracy of track prediction. In addition, the sector prediction result of each waypoint is listed in Table 2. Based on improving the accuracy of altitude prediction, the accuracy of sector prediction is also significantly improved. For air traffic control systems such as flow management system, traffic capacity assessment, route planning, and conflict resolution strategies can only be carried out by controllers based on knowing the expected overflight situation of sectors and information regions. Therefore, the method of optimizing track prediction model based on historical track data is of great significance for practical engineering application.
In order to further verify the effectiveness of historical data correction track prediction, this paper also counts the forecast and actual flight time of 100 flights from Beijing Airport to DPX route point. The waypoints from ZBAA to DPX are shown below.

(i) ZBAA

(ii) LADIX

(iii) IDKUP

(iv) P149

(v) PANKI

(vi) YQG

(vii) DALIM

(viii) ABTUB

(ix) P86

(x) P60

(xi) P58

(xii) UDINO

(xiii) DPX

Statistically, ZBAA-DPX has a flight time of about $3120 \mathrm{~s}$ based on the projected route before the analysis of historical data is used. Using the historical data analysis, obtaining the effective control experience altitude of the reporting point, and so on, the recalculated average predicted flight time is $3323 \mathrm{~s}$. The corresponding real flight time obtained by radar detection is $3423 \mathrm{~s}$. It can be seen that by using the method described in this paper, the prediction accuracy is improved obviously, and the prediction error is raised to less than 5\% of the total flight time.

In terms of computational efficiency, the traditional track prediction method based on data mining is too timeconsuming to meet the requirements of real-time calculation. For example, in [23], a total of 12080 tracks in 25 days are selected as the training set. After off-line learning, the actual radar data of different lengths are used as the prediction input, and the time consumption of a predicted track output by the four-dimensional track prediction algorithm based on FPR-Tree (Frequent Path-tree combined R-tree) is tested, and the results are shown in Figure 11. It can be seen that, with the increase of the number of input radar points, the time-consuming of the algorithm is also increasing; especially, when the input radar points exceed 100 points, the time-consuming increases by leaps and bounds. On the other hand, the method used in this paper obtains the experience data of control intention off-line based on the historical track 


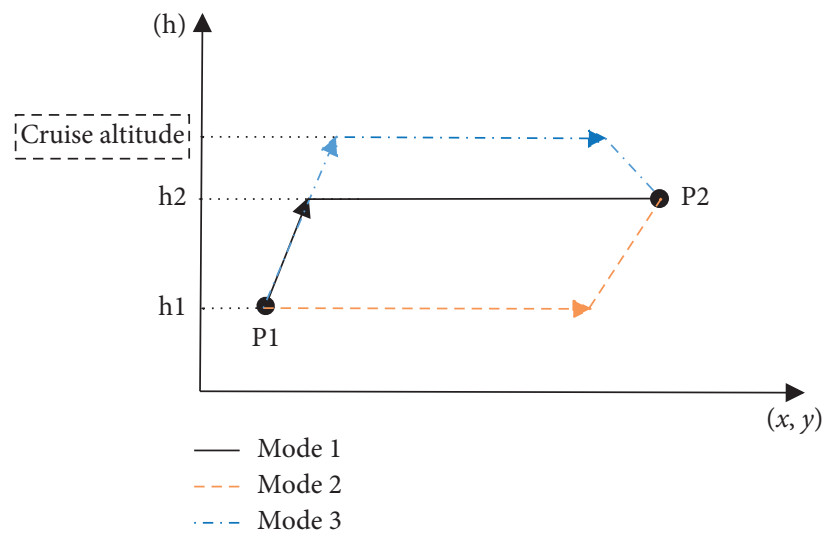

Figure 7: Schematic diagram of maneuver modes.

TABLE 1: Experience parameters by historical data mining.

\begin{tabular}{lccc}
\hline Point name & Longitude & Latitude & Height $(\mathrm{m})$ \\
\hline BISAL & 112.2269 & 37.1006 & 9500 \\
P130 & 112.4925 & 37.5442 & 9500 \\
XIVEP & 112.6578 & 37.6322 & 9500 \\
UBLAT & 113.4436 & 38.0503 & 9500 \\
ISGOD & 114.0897 & 38.285 & 8100 \\
AKLOL & 114.3994 & 38.3969 & 8100 \\
OC & 114.5558 & 38.4539 & 8100 \\
AVNIX & 116.075 & 38.7167 & 4500 \\
BOBAK & 116.4047 & 39.1264 & 4500 \\
Cruising altitude & $10100 \mathrm{~m}$ & Cruising velocity & $900 \mathrm{~m} / \mathrm{s}$ \\
\hline
\end{tabular}

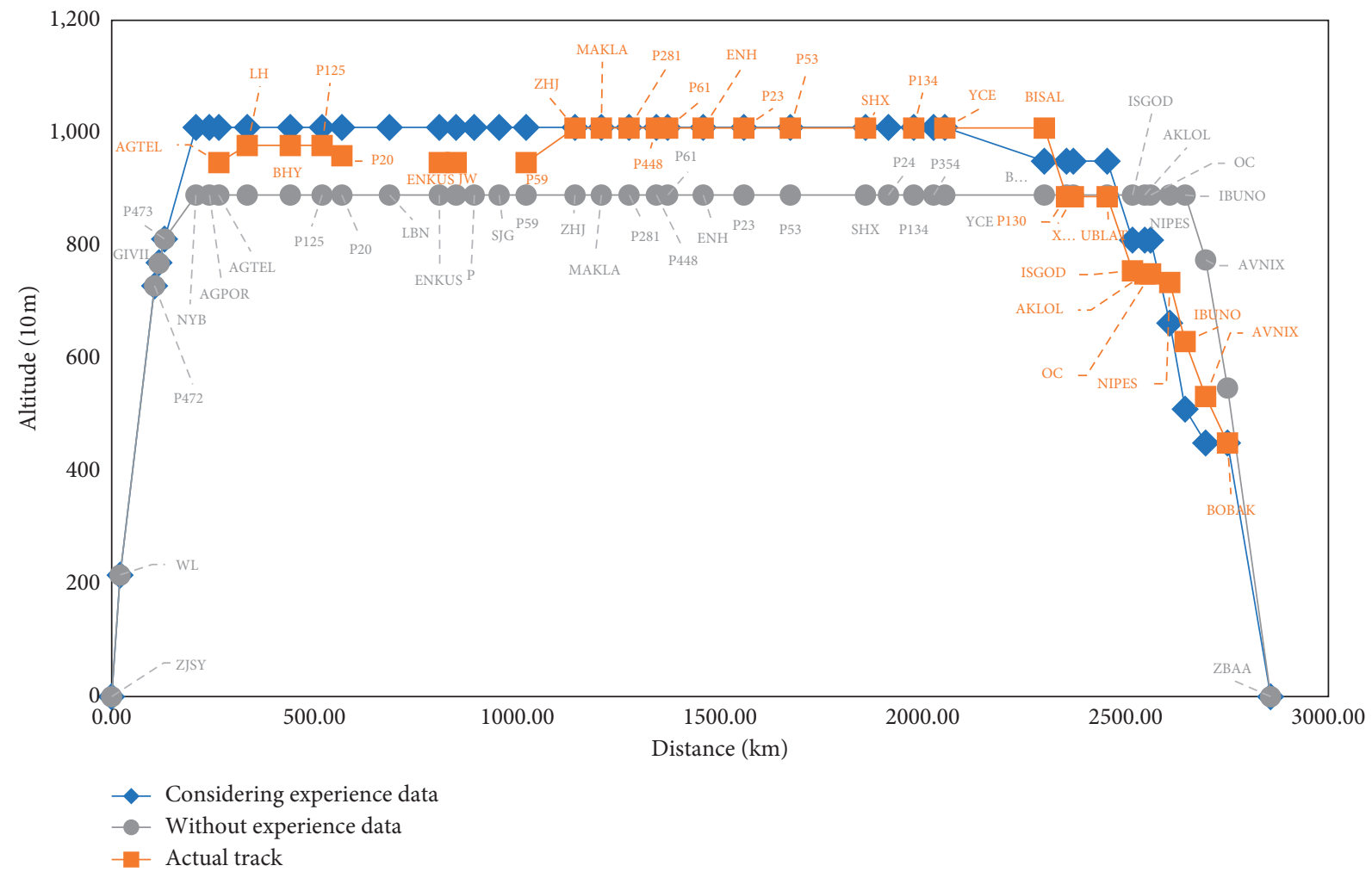

FIgURE 8: Curves of distance-altitude with this method. 


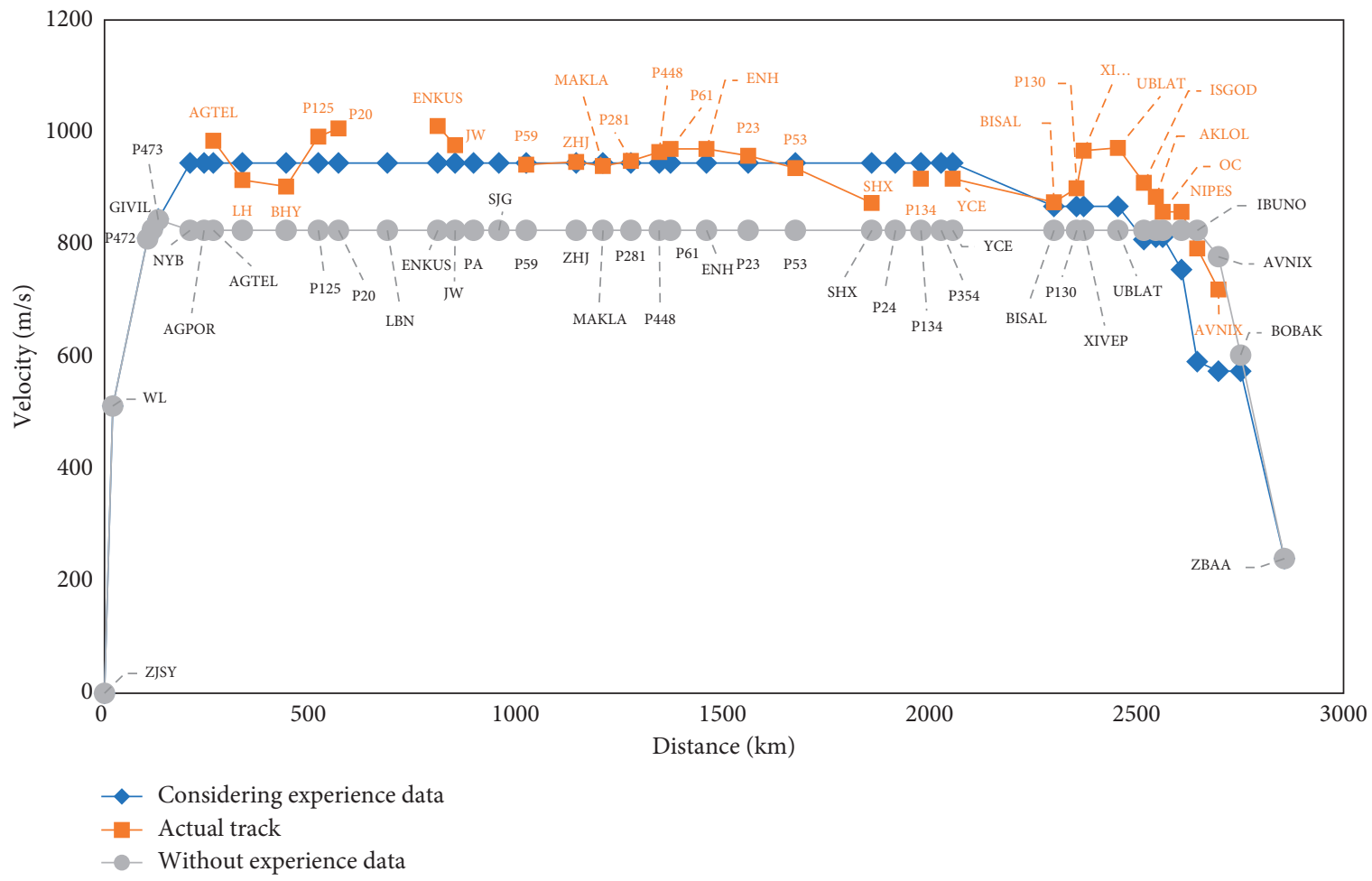

FIgURE 9: Curves of distance-velocity with this method.

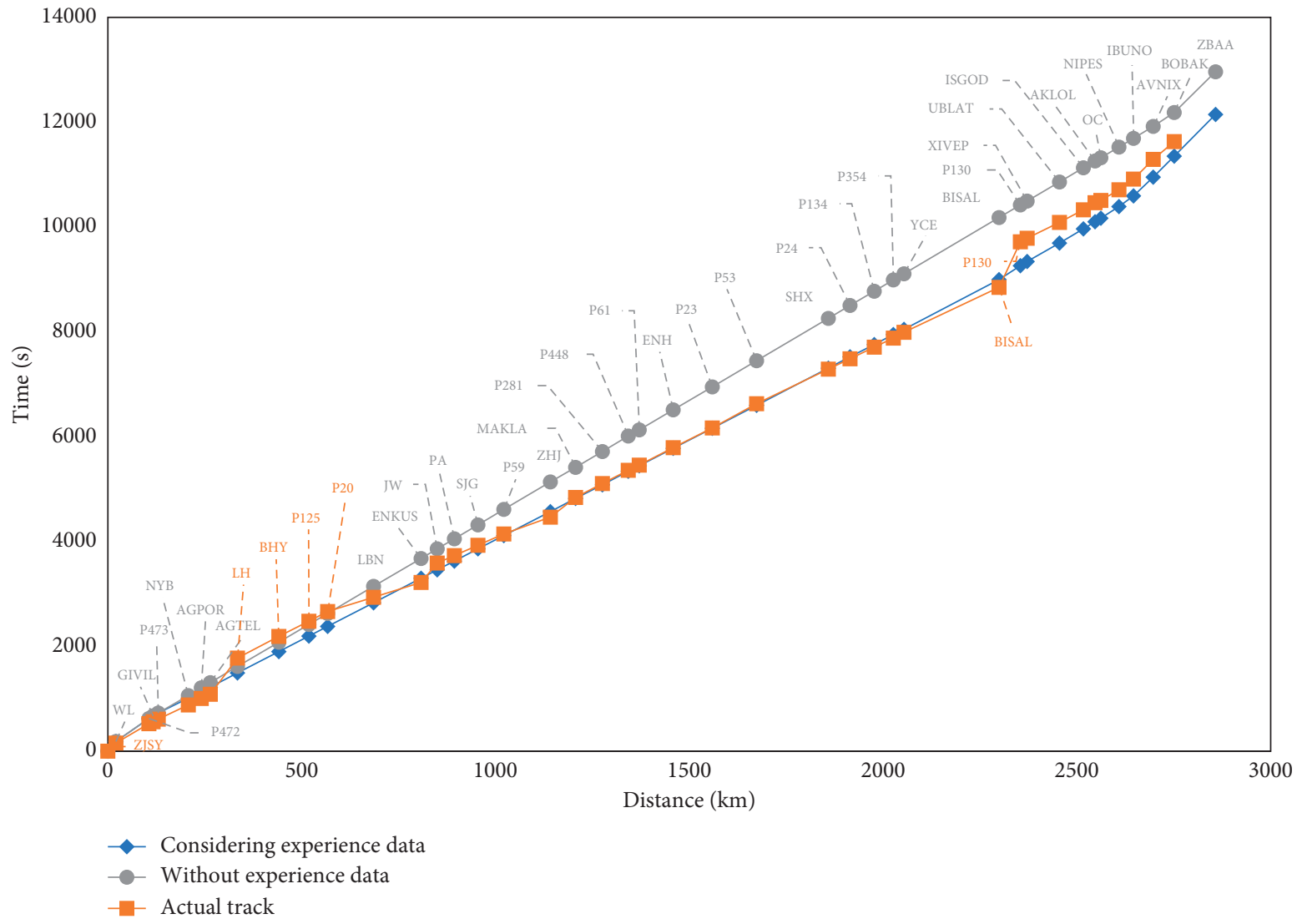

FIgURE 10: Curves of distance-time with this method. 


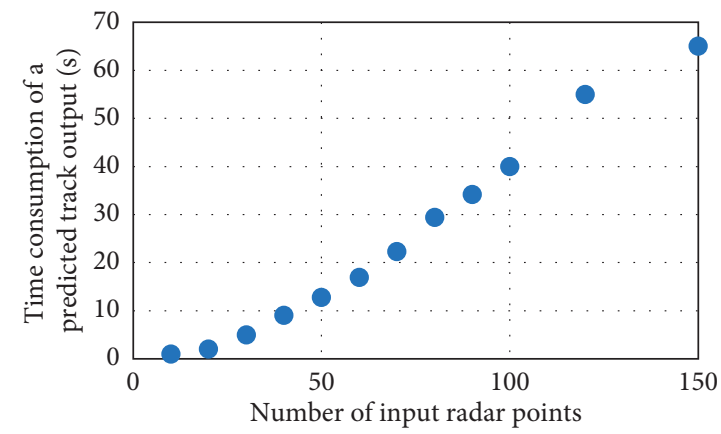

FIGURE 11: Time-consuming of track prediction based on the traditional data-mining method.

TABLE 2: Sheet of section prediction results with this method.

\begin{tabular}{|c|c|c|c|c|c|c|c|}
\hline $\begin{array}{l}\text { Point } \\
\text { name }\end{array}$ & $\begin{array}{c}\text { Without } \\
\text { experience data }\end{array}$ & $\begin{array}{c}\text { Considering } \\
\text { experience data }\end{array}$ & Real track & $\begin{array}{l}\text { Point } \\
\text { name }\end{array}$ & $\begin{array}{c}\text { Without } \\
\text { experience data }\end{array}$ & $\begin{array}{c}\text { Considering } \\
\text { experience data }\end{array}$ & Real track \\
\hline ZJSY & ZJSYAR04 & ZJSYAR04 & ZJSYAR04 & P61 & - & - & - \\
\hline WL & ZJSYAR04 & ZJSYAR04 & ZJSYAR04 & ENH & ZGGGAR17 & ZGGGAR17 & ZGGGAR17 \\
\hline P472 & ZJSYAR04 & ZJSYAR04 & ZJSYAR04 & P23 & ZGGGAR17 & ZGGGAR17 & ZGGGAR17 \\
\hline GIVIL & ZJSYAR04 & ZJSYAR04 & ZJSYAR04 & P53 & ZGGGAR17 & ZGGGAR17 & ZGGGAR17 \\
\hline P473 & ZJSYAR04 & ZJSYAR04 & ZJSYAR04 & SHX & ZLXYAR02 & ZLXYAR07 & ZLXYAR07 \\
\hline NYB & ZJSYAR04 & ZJSYAR01 & ZJSYAR01 & P24 & ZLXYAR02 & ZLXYAR07 & ZLXYAR07 \\
\hline AGPOR & ZJSYAR04 & ZJSYAR01 & ZJSYAR01 & $\mathrm{P} 134$ & $Z B A A A R 27$ & ZBAAAR18 & ZBAAAR18 \\
\hline AGTEL & ZJSYAR11 & ZJSYAR11 & ZJSYAR11 & P354 & $Z B A A A R 27$ & ZBAAAR18 & ZBAAAR18 \\
\hline $\mathrm{LH}$ & ZJSYAR11 & ZJSYAR11 & ZJSYAR11 & YCE & ZBAAAR08 & ZBAAAR08 & ZBAAAR08 \\
\hline $\mathrm{BHY}$ & ZGZJAR02 & ZJSYAR12 & ZJSYAR12 & BISAL & ZBAAAR08 & ZBAAAR08 & ZBAAAR08 \\
\hline P125 & ZGZJAR02 & ZJSYAR12 & ZJSYAR12 & $\mathrm{P} 130$ & ZBAAAR08 & ZBAAAR08 & ZBAAAR08 \\
\hline P20 & ZGNNAR01 & ZGNNAR04 & ZGNNAR01 & XIVEP & ZBAAAR07 & ZBAAAR07 & ZBAAAR07 \\
\hline LBN & ZGNNAR01 & ZGNNAR04 & ZGNNAR04 & UBLAT & ZBAAAR07 & ZBAAAR07 & ZBYNAR04 \\
\hline ENKUS & ZGNNAR01 & ZGNNAR04 & ZGNNAR01 & ISGOD & ZBAAAR07 & ZBAAAR07 & ZBYNAR04 \\
\hline JW & ZGGGAR22 & ZGGGAR11 & ZGGGAR11 & AKLOL & ZBAAAR05 & ZBAAAR05 & ZBAAAR05 \\
\hline PA & ZGGGAR22 & ZGGGAR11 & ZGGGAR11 & $\mathrm{OC}$ & ZBAAAR05 & ZBAAAR05 & ZBAAAR05 \\
\hline SJG & ZGGGAR22 & ZGGGAR11 & ZGGGAR11 & NIPES & ZBAAAR05 & ZBAAAR05 & ZBAAAR05 \\
\hline P59 & ZGGGAR22 & ZGGGAR11 & ZGGGAR11 & IBUNO & ZBAAAR05 & ZBAAAR05 & ZBAAAR05 \\
\hline $\mathrm{ZHJ}$ & ZGGGAR32 & ZGGGAR32 & ZGGGAR32 & AVNIX & ZBAAAR04 & ZBAAAR06 & ZBAAAR06 \\
\hline MAKLA & - & - & - & BOBAK & ZBAAAP06 & ZBAAAP06 & ZBAAAP06 \\
\hline P281 & - & - & - & ZBAA & ZBAAAP03 & ZBAAAP03 & ZBAAAP03 \\
\hline P448 & - & - & - & - & - & - & - \\
\hline
\end{tabular}

data and only inputs the experience values as the calculation parameters of the dynamic model. The calculation time level is just millisecond level, which can be ignored. Therefore, it can meet the requirements of real-time track prediction and correction and has obvious advantages.

\section{Conclusion}

The method in this paper is to use historical data to extract the experienced altitude and cruise altitude of the aircraft and combine them with the aircraft performance model and weather data to realize $4 \mathrm{D}$ track prediction. This method has the following advantages in the application of air traffic management systems such as traffic flow management and regional cooperative decision-making.

First of all, based on the method of extracting control intention from historical track data, the intention information data files, such as the altitude, cruise altitude, and vacuum speed, were generated for each reporting point under the condition of city pair and aircraft type field, and the control intention can be extracted quickly and procedurally.

Second, the $4 \mathrm{D}$ flight path prediction mathematicalphysical model is established, which is coupled with the aircraft performance model and control intention model. The prediction model takes into account many kinds of information, such as weather, control intention, and aircraft performance, which can quickly and accurately predict the $4 \mathrm{D}$ flight path of aircraft and improve the control efficiency.

Third, the strategy of using aircraft maneuvering mode is proposed. According to the relation between target point and current calculating report point, the trigger time of control command is simulated, and the optimization of the track prediction model based on the result of historical track data mining is realized.

Forth, the proposed method is verified by comparing the predicted flight time with the real transit time. The results show that compared with the real transit time, the average 
error of the $4 \mathrm{D}$ prediction method is less than $5 \%$ of the flight time. The optimization method of the track prediction model proposed in this paper is effective and reliable.

Fifth, the follow-up work can be based on the historical track data to mine the experience range and maneuver law of the flight segment to form a more perfect control intention sequence and explore the coupling method with the aircraft performance model to further improve the accuracy of the four-dimensional track prediction.

\section{Data Availability}

The data used to support the findings of this study are available from the corresponding author upon request.

\section{Conflicts of Interest}

The authors declare that they have no conflicts of interest.

\section{Acknowledgments}

This research was supported by the Natural Science Foundation for Youths of Jiangsu Province (Grant no. BK20180132).

\section{References}

[1] R. Nikhil and K. Gnana Sheela, "Intelligent air traffic control using neural networks," International Journal of Science Technology \& Engineering, vol. 2, no. 8, pp. 323-327, 2016.

[2] S. Ruiz, M. Piera, and I. Pozo, "A medium term conflict detection and resolution system for terminal maneuvering area based on spatial data and 4D trajectories," Transportation Research: Part C, vol. 26, pp. 296-417, 2013.

[3] G. Hancerliogullari, G. Rabadi, A. H. Al-Salem, and M. Kharbeche, "Greedy algorithms and metaheuristics for a multiple runway combined arrival-departure aircraft sequencing problem," Journal of Air Transport Management, vol. 32, pp. 39-48, 2013.

[4] C. A. Zúñiga, M. A. Piera, S. Ruiz, and I. Del Pozo, "A CD\&CR causal model based on path shortening/path stretching techniques," Transportation Research Part C: Emerging Technologies, vol. 33, pp. 238-256, 2013.

[5] J. Rosenow, H. Fricke, and M. Schultz, "Impact of multicriteria optimized trajectories on European airline efficiency, safety and airspace demand," Journal of Air Transport Management, vol. 78, pp. 133-143, 2019.

[6] J. Rosenow, H. Fricke, and M. Schultz, "Air traffic simulation with 4D multi-criteria optimized trajectories," in Proceedings of the 2017 Winter Simulation Conference (WSC), pp. 25892600, IEEE Xplore, Las Vegas, NV, USA, December 2017.

[7] S. K. Hong and K. J. Lee, "Trajectory prediction for vectored area navigation arrivals," Journal of Aerospace Information Systems, vol. 7, no. 12, pp. 1-13, 2015.

[8] R. Alligier and D. Gianazza, "Learning aircraft operational factors to improve aircraft climb prediction: a large scale multi-airport study," Transportation Research Part C: Emerging Technologies, vol. 96, pp. 72-95, 2018.

[9] S. Ayhan and H. Samet, "Aircraft trajectory prediction made easy with predictive analytics," in Proceedings of the 22nd ACM SIGKDD International Conference on Knowledge Discovery and Data Mining, pp. 21-30, Association for Computing Machinery, New York, NY, USA, August 2016.
[10] R. Alligier, D. Gianazza, and N. Durand, "Machine learning and mass estimation methods for ground-based aircraft climb prediction," IEEE Transactions on Intelligent Transportation Systems, vol. 6, no. 16, pp. 3138-3149, 2015.

[11] X. M. Tang, L. Zhou, and Z. Y. Shen, "Etc. 4D trajectory prediction of aircraft taxiing based on fitting velocity profile," Aeronautical Computing Technology, vol. 1, pp. 35-39, 2015.

[12] W. Schuster, "Trajectory prediction for future air traffic management - complex manoeuvres and taxiing," The Aeronautical Journal, vol. 119, no. 1212, pp. 121-143, 2015.

[13] W. B. Du and C. D. Wang, "Trajectory model and optimization based on path object," Computer Technology and Development, vol. 8, no. 22, pp. 249-252, 2012.

[14] M. Kaiser, M. Schultz, and H. Fricke, Enhanced Jet Performance Model for High Precision 4D Flight Path Prediction, ATACCS, Barcelona, Spain, 2011.

[15] U. Mevlut, G. Gunney, and K. Emre, Flight Deck Centered Tactical 4D Trajectory Planning and Collision Avoidance with Flight Envelope Sampling, ATACCS, Toulouse, France, 2015.

[16] Z. Y. Zhao, W. L. Zeng, and Z. B. Quan, "Etc. Aircraft trajectory prediction using deep long short-term memory networks," in Proceedings of the 19th COTA International Conference of Transportation Professionals, pp. 2330-2341, Southeast University, Nanjing, China, July 2019.

[17] W. Zeng, Z. Quan, Z. Zhao, C. Xie, and X. Lu, "A deep learning approach for aircraft trajectory prediction in terminal airspace," IEEE Access, vol. 8, pp. 151250-151266, 2020.

[18] M. Gariel, A. Srivastava, and E. Feron, "Trajectory clustering and an application to airspace monitoring," Intelligent Transportation Systems, vol. 4, no. 12, pp. 1511-1524, 2011.

[19] E. L. Zhao, W. N. Hao, and F. Zhao, "Improved track clustering algorithm based on density," Computer Engineering, vol. 37, no. 9, pp. 270-272, 2011.

[20] K. Qian, Y. Zhou, and J. Yang, "Aircraft target track prediction model based on BP neural network," Command Information System and Technology, vol. 3, no. 8, pp. 54-58, 2017.

[21] X. M. Tang, J. W. Gu, and Z. Y. Shen, "A flight profile clustering method combining twed with K-Means algorithm for 4D trajectory prediction," in Proceedings of the 2015 Integrated Communication, Navigation, and Surveillance Conference, IEEE Xplore, HerQdon, Virginia, USA, April 2015.

[22] S. Y. Jiang, G. Chen, and J. Tao, "Study on 4D track prediction and optimization of aircraft climbing phase," in Proceedings of the 2019 International Conference on Communications, Information System and Computer Engineering (CISCE), pp. 456-460, IEEE, Haikou, China, July 2019.

[23] Y. Ma, Research on Precise Prediction Method of 4D Trajectory Based on Data Mining, Nanjing University of Aeronautics and Astronautics The Graduate School, Nanjing, China, 2016. 